\title{
Anatomía de la semilla de cuatro especies mexicanas de Hippocratea (Celastraceae)
}

\author{
MA. GUADALUPE ESPINOSA-OSORNIO y E. MARK ENGLEMAN ${ }^{1}$
}

REsumen. Se estudió la morfología y, mediante microscopía fotónica, la anatomía de semillas maduras de $H$. acapulcensis, $H$. celastroides, $H$. excelsa y $H$. volubilis. En las cuatro especies las semillas son aladas y el ala es mas grande que el cuerpo que contiene el embrión. El ala puede ser membranosa o casi toda engrosada por la abundancia de células traqueoidales isodiamétricas. El haz vascular de la rafe recorre el ala longitudinalmente en la parte media; además un haz de células traqueoidales alargadas recorre por uno de los bordes del ala, o cerca de éste. El ala tiene también zonas engrosadas por la abundancia de células traqueoidales isodiamétricas de paredes gruesas. El embrión es grande con un eje pequeño. Los cotiledones son connados y pueden o no conservar la epidermis en la superficie adaxial, pero una cutícula sólo se observó en la superficie abaxial. La cubierta seminal se forma de exotesta con taninos, mesotesta de células traqueoidales alargadas y endotegmen taninífero. Hay dos cutículas gruesas, la externa y la nucelar. Hay de uno a cuatro estratos de endospermo.

ABSTRACT. The morphology and anatomy of mature seeds of H. acapulcensis, $H$. celastroides, $H$. excelsa y $H$. volubilis were studied by light microscopy. In all four species the seeds are winged and the wing is larger than the body that contains the embryo. The wing may be mostly membranous or mostly thickened by the abundance of isodiametric tracheoidal cells. The vascular bundle of the raphe passes longitudinally through the middle of the wing. Additionally, a bundle of elongate tracheoidal cells passes along one edge of the wing or close by it. The wing has zones thickened by an abundance of thick-walled isodiametric tracheoidal cells. The embryo is large with a short axis. The cotiledons are connate and may or may not maintain the epidermis of the adaxial surface, but cuticle was observed only on the abaxial surface. The seed coat is formed by an exotesta with tannins, a mesotesta of elongate tracheoidal cells and a tanniniferous endotegmen. There are two thick cuticles, one on the exotesta and one on the nucellus. There are from one to four layers of endosperm.

${ }^{1}$ Centro de Botánica, Colegio de Postgraduados, 56230 Montecillo, Edo. de México.

Espinosa-Osornio MG, Engleman EM. 1994. Anatomía de la semilla de cuatro especies mexicanas de Hippocratea (Celastraceae). Boletín de la Sociedad Botánica de México 54: 57-67. 
El género Hippocratea cuenta con 100 especies, y diez de ellas se encuentran en México: $H$. acapulcensis, $H$. acutifolia, $H$.celastroides, $H$. chiapanensis, $H$. elliptica, $H$. excelsa, $H$. rovirosae, $H$. uniflora, $H$. utilis y $H$. volubilis. Estas se distribuyen en la costa del Pacífico desde Sinaloa hasta Chiapas, en la costa del Golfo desde Tamaulipas hasta Yucatán, y en Durango, Morelos, Puebla y Edo. de México (Standley, 1923; Palacios et al., 1989).

Algunas especies de Hippocratea poseen metabolitos secundarios que confieren propiedades terapeúticas e insecticidas. Hippocratea sp. permite un buen combate del gorgojo del maiz (Sitophilus zeamais Coleoptera: Curculionidae) y del barrenador mayor de los granos (Phostephanus truncatus Coleoptera: Bostrichidae) en maiz almacenado (Páez, 1987).H. excelsa, conocida como "cancerina", tiene efecto insecticida sobre plagas como S. zeamais, Tribolium castaneum (Coleoptera: Tenebrionidae), T. confusum (Coleoptera: Tenebrionidae), Rhizopertha dominica (Coleoptera: Bostrichidae), Cryptolestes sp. (Coleoptera: Cucujidae) y Gnathocerus maxillosos (Coleoptera: Tenebrionidae) en maíz almacenado (Torres, 1989). H. acapulcensis e H. celastroides son conocidas en algunos lugares de Guerrero, Michoacán y Oaxaca como "semilla del piojo" o "mata piojo", debido a sus propiedades insecticidas (Standley, 1923). Además, la cancerina se ha utilizado desde tiempos remotos en comunidades indígenas como planta medicinal, para ciertos padecimientos de la piel, úlceras gástricas y enfermedades del riñón (Palacios et al., 1989; Mata et al., 1990).

El estudio de la embriología de Hippocratea se restringe a seis especies: $H$. cinerascens, $H$. clematioides y $H$. grisebachii (Mauritzon, 1936), $H$. indica (David, 1938), H. grahamii (Adatia y Gavde, 1962; Johri, 1963) y H. celastroides (Espinosa-Osornio y Engleman, 1994); solo ésta última se distribuye en México. Adatia y Gavde (1962), con base en el estudio embriológico de $H$. grahamii, apoyan la separación del género en una familia aparte, la Hippocrateaceae. Al estudiar $H$. celastroides, nos dimos cuenta de que hay variabilidad en algunas características embriológicas del género; los óvulos pueden ser tenuinucelados o crasinucelados y el micrópilo puede estar formado por uno o dos tegumentos. Las seis especies estudiadas presentan características comunes como: a) óvulos anátropos, bitégmicos y con un tapete tegumentario; b) el desarrollo del saco embrionario de tipo Polygonum; y c) el desarrollo del endospermo de tipo nuclear (Davis, 1966).

Sin embargo, poco se indica sobre las características de las semillas maduras Adatia y Gavde (1962) mencionan que la cubierta seminal madura se forma del tegumento externo y del estrato más interno del tegumento interno. Corner (1976) sólo menciona que las semillas son exariladas y exalbuminosas. Hutchinson (1979) y Cronquist (1981) mencionan que las semillas son aladas, con una radícula diminuta, exalbuminosas y frecuentemente con los cotiledones connados. Espinosa-Osornio y Engleman (1993) describen detalladamente la cubierta seminal madura de 
H. celastroides. En este trabajo se plantea el estudio morfológico y anatómico de la semilla madura de cuatro especies de Hippocratea que se distribuyen en México, para ampliar el conocimiento sobre el género y para determinar si las características de la semilla madura presentan variabilidad intragenérica.

\section{MATERIALES Y MÉTODOS}

Se estudiaron semillas maduras de $H$. acapulcensis H.B.K. donadas por los herbarios MEXU y ENCEB, H. celastroides H.B.K. colectadas por los autores en el Estado de Morelos, H. excelsa H.B.K. donadas por el herbario MEXU y H. volubilis L. donadas por el herbario ENCEB (cuadro 1). A las semillas se les revisó primero su morfología externa; posteriormente se incluyeron en parafina para revisar su anatomía mediante microscopía fotónica. Se realizaron cortes de 10 micrómetros $(\mu \mathrm{m})$ en un microtomo rotatorio. Se tiñeron con safranina-verde fijo, rojo $\mathrm{O}$ de aceite y azul de toluidina-cloruro de calcio (Herr, 1992).

CUADRO 1. Fechas y localidades de colecta de las semillas de Hippocratea spp.

\begin{tabular}{|l|l|l|l|l|l|}
\hline ESPECIE & HERBARIO & COLECTOR & REGISTRO & $\begin{array}{l}\text { FECHA } \\
\text { DE COLECTA }\end{array}$ & $\begin{array}{l}\text { LUGAR } \\
\text { DE COLECTA }\end{array}$ \\
\hline H. acapulcensis & MEXU & M. Quintani lla & S/N & abril 1981 & $\begin{array}{l}\text { Pico de Oro, } \\
\text { Chis. } \\
\text { Jojutla Mor. }\end{array}$ \\
\hline H. celastroides & CHAPA & $\begin{array}{l}\text { Espinosa-Osornin } \\
\text { y Engleman }\end{array}$ & S/N & marzo 1991 & $\begin{array}{l}\text { Tequesquitengo, } \\
\text { Mor. }\end{array}$ \\
\hline H. excelsa & MEXU & E. Matuda & 31223 & julio 1922 & $\begin{array}{l}\text { San Antonio } \\
\text { Tlataya, Edo. } \\
\text { de México }\end{array}$ \\
\hline H. volubilis & ENCEB & ---- & --- & marzo 1972 & $\begin{array}{l}\text { Ocozocuatla de } \\
\text { Espinosa }\end{array}$ \\
\hline
\end{tabular}




\section{RESULTADOS}

Morfología externa. Las semillas de las cuatro especies son aladas. La semilla de $H$. celastroides mide $42 \mathrm{~mm}$, la de $H$. acapulcensis $36 \mathrm{~mm}, H$. excelsa $35 \mathrm{~mm}$ y $H$. volubilis alcanza sólo unos $29 \mathrm{~mm}$. En todos los casos el ala es mayor que el cuerpo que contiene el embrión (fig. 1). En las cuatro especies, el haz vascular de la rafe recorre longitudinalmente la parte media del ala. En $H$. acapulcensis y $H$.celastroides el ala es membranosa y en el borde ventral tiene un haz de células traqueoidales. En las cuatro especies el ala tiene amplias zonas engrosadas debido a la presencia de muchas células traqueoidales. La forma y tamaño de estas zonas varían dependiendo de la especie. En H.acapulcensis es apenas una línea en el borde dorsal. En H. celastroides es una zona pequeña en el borde dorsal. En H.volubilis ocupa casi el $50 \%$ de la superficie del ala y se encuentra a ambos lados del haz vascular de la rafe. En H. excelsa esta zona es aún más extensa; ocupa casi el 100\% de la superficie entre el haz vascular de la rafe y el haz de células traqueoidales, y casi el $50 \%$ de la superficie de la otra mitad del ala. El ángulo que la cubierta forma en la cercanía del micrópilo es poco menor de $90^{\circ}$ en $H$. acapulcensis y H.celastroides, es mayor a $90^{\circ}$ en $H$. volubilis y es de casi $180^{\circ}$ en $H$. excelsa.
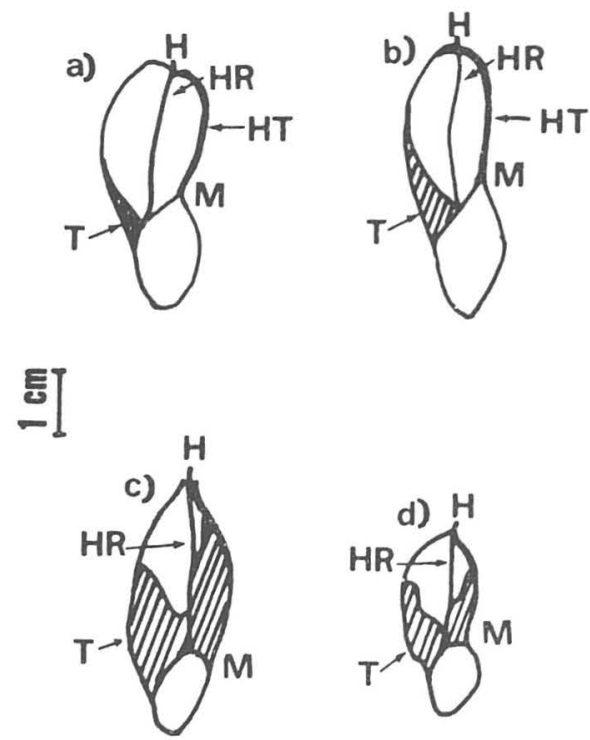

Fig. 1. Semillas de cuatro especies de Hippocratea. a) H. acapulcensis. b) H. celastroides. c) H. excelsa. d) H. volubilis. $\mathrm{T}=$ zona engrosada con células traqueoidales; $\mathrm{HT}=$ haz de células traqueoidales; $\mathrm{HR}=$ haz vascular de la rafe; $\mathrm{H}=$ hilo; $\mathrm{M}=$ zona del micrópilo. 
CUADRO 2. Longitud del embrión y eje embrionario en Hippocratea spp. (promedios de 3-10 mediciones).

\begin{tabular}{|l|c|c|c|}
\hline ESPECIE & $\begin{array}{c}\text { EMBRIÓN } \\
(\mathrm{mm})\end{array}$ & $\begin{array}{c}\text { EJE EMBRIONARIO } \\
(\mathrm{mm})\end{array}$ & $\begin{array}{c}\text { PROPORCIÓN } \\
\text { EJE-EMBRIÓN }\end{array}$ \\
\hline H. acapulcensis & 15.5 & 1.3 & 0.084 \\
H. celastroides & 18.4 & 1.0 & 0.054 \\
H. excelsa & 13.1 & 1.0 & 0.076 \\
H. volubilis & 10.8 & 0.9 & 0.083 \\
\hline
\end{tabular}

Anatomía del embrión. El embrión es grande, con un eje pequeño (cuadro 2) Ocupa el $8.4 \%$ del total de la longitud del embrión en $H$. acapulcensis, $7.6 \%$ en $H$. excelsa y $8.3 \%$ en $H$. volubilis. $H$. celastroides es la especie que proporcionalmente tiene el eje embrionario mas pequeño; ocupa sólo el 5.4\% del embrión. Los cotiledones son connados. En $H$. acapulcensis y $H$. celastroides no hay epidermis intercotiledonar; la epidermis se recorre sólo dos o tres células entre los bordes de los cotiledones, pero la cutícula del lado abaxial no entra en la zona de contacto de los lados adaxiales (figs. 2 a y $2 \mathrm{~b}) . H$. volubilis sí conserva las epidermis intercotiledonares, pero la cutícula sólo cubre las primeras tres o cuatro células periféricas de la unión (fig. 2d), mientras que en $H$. excelsa hay epidermis intercotiledonar y una delgada cutícula cubre las primeras 12-15 células de la epidermis intercotiledonar (fig. 2c). La epidermis de la superficie abaxial de los cotiledones, en todos los casos se halla cubierta por una delgada cutícula (línea gruesa en la fig. 2). En las cuatro especies se observaron en los cotiledones drusas con un tamaño máximo de $16 \mu \mathrm{m}$. En $H$. volubilis los cotiledones desarrollaron abundantes espacios intercelulares, al parecer esquizógenos. En $H$. excelsa existen espacios similares pero en menor cantidad, mientras que en $H$. acapulcensis e $H$. celastroides los cotiledones son compactos.

Cubierta seminal. La cubierta seminal en las cuatro especies se forma por la exotesta, la mesotesta y el endotegmen. Las cutículas que se observaron fueron la externa y la nucelar. En todos los casos la cutícula externa es más gruesa que la nucelar, pero ambas son bastante más gruesas que la cutícula cotiledonar. En $H$. celastroides se observó algunas veces una delgada cutícula intertegumentaria. El grosor de la cubierta varía con la especie (cuadro 3). La exotesta es taninífera; sólo en $H$. acapulcensis la exotesta tiene pocos taninos. La mesotesta tiene células traqueoidales. El endotegmen es taninifero, pero en $H$. excelsa los taninos son poco evidentes (fig. 3). La forma de las células traqueoidales de la cubierta es siempre alargada, pero varía 
CUADRO 3. Grosores de la cubierta de Hippocratea spp. en micrómetros (promedios de 15 mediciones).

\begin{tabular}{|l|c|c|c|c|}
\hline ESPECIE & CUBIERTA & EXOTESTA & MESOTESTA & ENDOTEGMEN \\
\hline H. acapulcensis & 151 & 21 & 125 & 5 \\
H. celastroides & 164 & 35 & 120 & 9 \\
H. excelsa & 116 & 49 & 64 & 3 \\
H. volubilis & 120 & 28 & 77 & 15 \\
\hline
\end{tabular}

el grosor y la forma de los engrosamientos de la pared lignificada. En H. acapulcensis la pared de las células traqueoidales se tiñe fuertemente con safranina y con azul de toluidina-cloruro de calcio; la mayor parte de la pared está lignificada y presenta puntuaciones. En $H$. celastroides la pared de las células traqueoidales puede tener puntuaciones o engrosamientos reticulados o escalariformes, pero en los tres casos la pared se tiñe fuertemente con safranina y azul de toluidina-cloruro de calcio. En $H$. excelsa la tinción con safranina no es fuerte en toda la cubierta; los estratos subyacentes a la exotesta se tiñen más que los estratos más internos, en parte por que las paredes son más gruesas en la periferia. Con azul de toluidina-cloruro de calcio la tinción es

a)
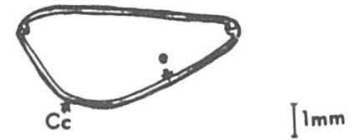

b)

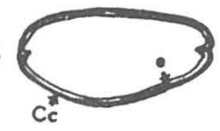

c)

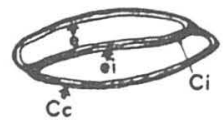

d)

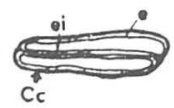

Fig. 2. Corte transversal del embrión al nivel de los cotiledones, mostrando cutícula y epidermis. a) $H$. acapulcensis; b) $H$. celastroides; c) $H$. excelsa; d) $H$. volubilis. e= epidermis cotiledonar abaxial; ei= epidermis intercotiledonar, $\mathrm{Cc}=$ cutícula cotiledonar, $\mathrm{Ci}=$ cutícula intercotiledonar. 
A

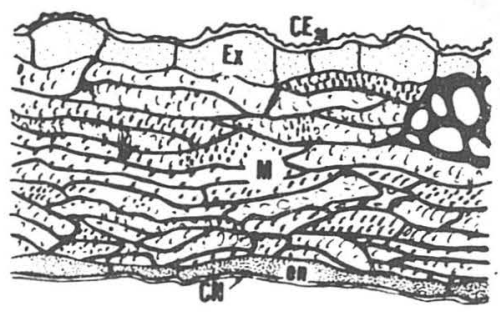

C

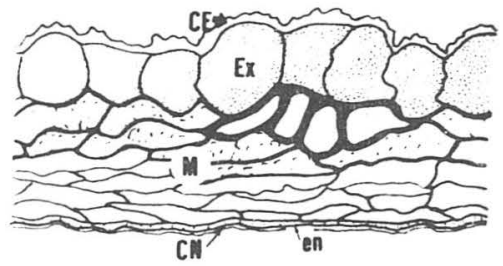

B

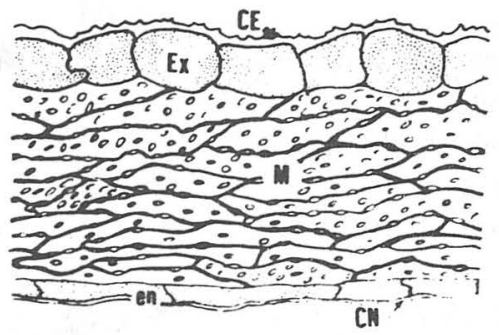

D

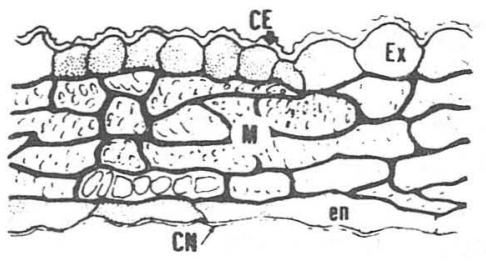

Fig. 3. Cubierta seminal. a) H. acapulcensis; b) H. celastroides; c) H. excelsa; d) H. volubilis. $\mathrm{Ex}=$ exotesta; $\mathrm{M}=$ mesotesta; $\mathrm{en}=$ endotegmen; $\mathrm{CE}=$ cutícula externa; $\mathrm{CN}=$ cutícula nucelar, $\mathrm{CI}=$ cutícula intertegumentaria. La barra equivale a $50 \mu \mathrm{m}$.

intensa en los estratos periféricos, pero la región interna de la mesotesta no se tiñe, lo cual indica que sus paredes no están lignificadas. Los engrosamientos son reticulados y escalariformes, pero muchas células son colapsadas. En $H$. volubilis las células traqueoidales tiñen fuertemente con safranina y con azul de toluidina. Las paredes son gruesas con engrosamientos reticulados. El grosor de las paredes va de mayor a menor en $H$. acapulcensis, $H$. celastroides, $H$. volubilis y $H$. excelsa

Endospermo. Debajo de la cubierta seminal, se encontraron de uno a cuatro estratos de endospermo (cuadro 4).

Cuadro 4. Endospermo en Hippocratea spp.

\begin{tabular}{|l|c|c|}
\hline Especies & Número de estratos & $\begin{array}{c}\text { Grosor }(\mu \mathrm{m}) \\
\text { (promedio de } 15 \text { mediciones) }\end{array}$ \\
\hline H. acapulcensis & $1-3$ & 28 \\
H. celastroides & $2-3$ & 20 \\
H. excelsa & $2-3$ & 42 \\
H. volubilis & $3-4$ & 40 \\
\hline
\end{tabular}




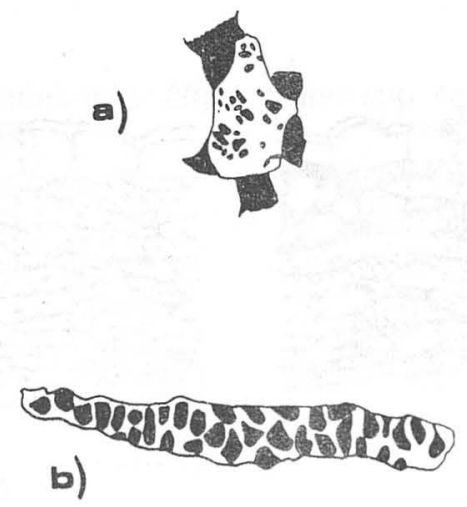

Fig 4. Células traqueoidales en $H$. celastroides. a) ala; b) cubierta.

Ala. En cortes transversales se pudo observar que el ala está cubierta de una epidermis como la de la cubierta del cuerpo de la semilla. EL interior del ala está compuesto de: a) células de pared delgada sin engrosamientos y b) células de pared engrosada y lignificada (células traqueoidales). Las células traqueoidales del ala difieren de las de la cubierta seminal en que son casi isodiamétricas, tienen pocas puntuaciones y pueden presentar espacios intercelulares grandes (fig. 4). En las cuatro especies el haz vascular de la rafe es el único y atraviesa la parte media del ala. En la figura 5 se muestran cortes transversales del ala al nivel medio de la semilla completa.

En $H$. acapulcensis y $H$. celastroides casi toda el ala es membranosa, la zona engrosada es pequeña; la parte membranosa del ala es más gruesa en la zona entre la rafe y el haz de células traqueoidales que del otro lado de la rafe, y mide de 59 a 97 $\mu \mathrm{m}$. En el borde dorsal se encuentra la pequeña zona engrosada de células traqueoidales en donde el grosor del ala alcanza hasta $208 \mu \mathrm{m}$. La parte membranosa del ala puede o no tener células con paredes lignificadas. En ocasiones las células epidérmicas y subepidérmicas tienen paredes lignificadas y las células más internas conservan sus paredes delgadas. El haz de células traqueoidales se ubica en el borde ventral del ala y tiene de 277 a $515 \mu \mathrm{m}$ de grosor. En $H$. excelsa las células de los dos o tres estratos subepidérmicos tienen paredes lignificadas y las células más internas conservan sus paredes delgadas. En $H$. volubilis las células tienen paredes lignificadas, pero una pequeña parte del ala, las células más internas en corte transversal, sobre todo entre el haz vascular y el haz de células traqueoidales conservan sus paredes delgadas.

En $H$. excelsa y $H$. volubilis la mayor parte del ala es engrosada, alcanzando de 762 a $1079 \mu \mathrm{m}$ de grosor en la zona del haz vascular de la rafe. El haz de células traqueoidales no se encuentra hasta la orilla del ala y sus células tienen la paredes mas gruesas que las células traqueoidales que las rodean. Este haz no es vascular porque las células no se ordenan en tubos, ni hay presencia de floema. 


\section{DISCUSIÓN}

De acuerdo con Adatia y Gavde (1962), la cubierta de la semilla madura se forma de tegumento externo (exotesta y mesotesta) y el estrato más interno del tegumento interno (endotegmen). Nosotros observamos además que la exotesta y el endotegmen presentan taninos, aunque su concentración varía entre especies, y que la mesotesta está siempre formada por células traqueoidales. La mesotesta forma la mayor parte del grosor de la cubierta, del 57 al $83 \%$ en las cuatro especies.

En H. excelsa es posible que las semillas utilizadas hayan sido colectadas antes de su madurez, puesto que fueron colectadas en el mes de julio en San Antonio Tlataya, Edo. de México. Sabemos que $H$. celastroides se madura en febrero y marzo y que el último proceso en la maduración de la semilla es la lignificación de las paredes de la mesotesta, lo cual ocurre primero en las células externas. En las semillas examinadas de $H$. excelsa las células internas de la mesotesta no están lignificadas, tal vez por falta de madurez.

a)
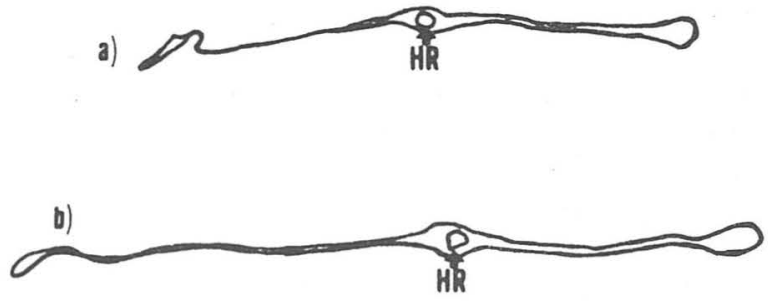

c)
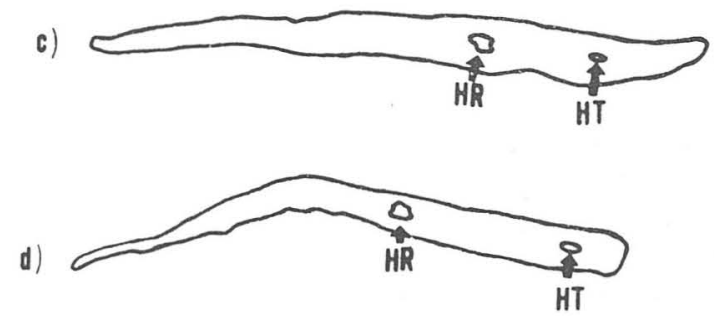

Fig. 5. Cortes transversales de ala, al nivel medio de la semilla completa. a) $H$. acapulcensis; b) $H$. celastroides; c) $H$. excelsa; d) $H$. volubilis. $\mathrm{HR}=$ haz vascular de la rafe; $\mathrm{HT}=$ haz de células traqueoidales. 
Con respecto al endospermo, Corner (1976), Hutchinson (1979) y Cronquist (1981) mencionan que no hay endospermo, pero aquí se encontró que siempre hay una cantidad pequeña de endospermo presente. En lo que se refiere al ala. aquí se observó que tanto la parte membranosa como la engrosada pueden estar constituídas por células de pared delgada o por células traqueoidales.

El eje del embrión es pequeño, tal y como lo mencionan Hutchinson (1979) y Cronquist (1981). Estos mismos autores mencionan que en el género Hippocratea los cotiledones frecuentemente se encuentran connados, pero no mencionan la condición anatómica. Aquí se encontró que las epidermis de la zona connada pueden estar presentes, como en $H$. excelsa y $H$. volubilis o ausente como en $H$. acapulcensis y $H$. celastroides, pero no se encontró cutícula en dicha zona.

\section{LITERATURA CITADA}

Adatia, R.D. y S.G. Gavde. 1962. Embryology of Celastraceac. En: Council of Scientific and Industrial Research. Plant embryology. A symposium. New Delhi. India. pp. $1-11$.

Corner, E.J.H. 1976. The seeds of dicotyledons. Cambridge University Press, London. 2 v.

Cronguist, A. 1981. A integrated system of classification of flowering plants. Columbia Univ. Press, New York.

DAVID. E. 1938. Embryologische Untersuchungen an Myoporaceen. Salvadoraceen. Sapindaceen und Hippocrateaceen. Planta 28:680-703.

Davis. G. L. 1966. Systematic embryology of the angiosperms. Wiley. New York. 528p.

Espinosa-Osornio. M.G. y E.M. Engil.m.MAn. 1993. Anatomía del desarrollo de la scmilla de Hippocratea celastroides H.B.K. Bol. Soc. Bot. México 53: 43-53.

HERR, J.M. 1992. New uses for calcium chloride solution as mounting medium. Biotcch. and Histochem. 67(1):9-13.

HutCHINSON, J. 1979. The families of flowering plants. Arranged according to a ncw system based on their phylogeny. Otto Kocltz Science Publishers, Germany. 968p.

JoHRI, B.M. 1963. Embryology and Taxonomy. En: P. Maheswari (Ed.). Recent advances in the embryology of angiosperms. Catholic Press. New Delhi. pp. 396-444.

Mata, R.. F. CAl/AdDA. E. Diaz y R.A. Toscano. 199(). Chemical studies on Mcxican plants used in traditional medicine. XV. Sesquiterpene evoniotate alkaloids from Hippocratea excelsa. J. Nat. Prod. 53(5):1212-1219.

Mauritzon, J. 1936. Embryologische Angaben über Stackousiaccac. Hippocrateaceac und Icacinaceae. Svensk. Bot. Tidskr. 30:541-550.

Palacios, J., R. Mata, R. Lópl\%. E. Linarlis, y R. BYi. 1989. Ilippocratea excelsa (Hippocrateaceac) a new source of transisoprenc. Econ. Bot. 43(4):5(18-5(1). 
PÁEZ L.. A. 1987. El uso de polvos vegetales e inertes minerales como una alternativa para el control del gorgojo del maíz Sitophilus zaemais Motschulsky (Coleoptera: Curculionidae) en maíz almacenado. Tesis de Maestría en Ciencias. Centro de Entomología y Acarología. Colegio de Postgraduados. Chapingo, México. 120p.

Standley, P.C. 1923. 83. Celastraceae. Bittersweet family. En: Trees and shrubs of Mexico. Contr. U. St. Natl. Herbr. 23(Part 3):676-685.

Torres R., J.C., 1989. Actividad de cuatro polvos minerales de Hippocratea excelsa en el combate de insectos plaga en maízalmacenado en Cárdenas, Tabasco. Tesis Ingeniero Agrónomo. Universidad Autónoma de Chapingo, México. 88p. 